\title{
UCL+Sheffield at SemEval-2016 Task 8: Imitation learning for AMR parsing with an $\alpha$-bound
}

\author{
James Goodman* Andreas Vlachos \# Jason Naradowsky* \\ * Computer Science Department, University College London \\ james@janigo.co.uk, jason.naradegmail.com \\ \# Department of Computer Science, University of Sheffield \\ a.vlachos@sheffield.ac.uk
}

\begin{abstract}
We develop a novel transition-based parsing algorithm for the abstract meaning representation parsing task using exact imitation learning, in which the parser learns a statistical model by imitating the actions of an expert on the training data. We then use the imitation learning algorithm DAGGER to improve the performance, and apply an $\alpha$-bound as a simple noise reduction technique. Our performance on the test set was $60 \%$ in F-score, and the performance gains on the development set due to DAgGer was up to 1.1 points of Fscore. The $\alpha$-bound improved performance by up to 1.8 points.
\end{abstract}

\section{Introduction}

In abstract meaning representation parsing (Banarescu et al., 2013), the goal is to parse natural language in a domain-independent graph-based meaning representation (AMR). In the first AMR parsing work, Flanigan et al. (2014) split the task into two sub-tasks; concept identification and graph creation. The sub-tasks are learned independently, and exact inference is used to find highest-scoring maximum spanning connected acyclic graph that contains all the concepts identified in the first stage. Later work by Wang et al. (2015b) adopted a different strategy based on the similarity between the dependency parse of a sentence and the semantic AMR graph. They start from the dependency parse and learn a transition-based parser that converts it into an AMR graph. To learn the parser, Wang et al. (2015b) define an algorithm that for each instance in the training data infers the action sequence that convert the input dependency tree into the corresponding AMR graph and train a classifier to predict the actions to be taken during testing. This strategy is also referred to as exact imitation learning, while the algorithm that infers the action sequence in the training instances is commonly referred to as the expert policy.

In our submission to SemEval Task 8 on AMR parsing, we follow the transition-based paradigm of Wang et al. (2015b) with modifications to the parsing algorithm, and also use the DAGGER imitation learning algorithm (Ross et al., 2011) to generalise better to unseen data. The central idea of DAGGER is that the distribution of states encountered by the expert policy during training may not be a good approximation to those seen in testing by the trained policy. Previous work by Rao et al. (2015) used SEARN, a similar imitation learning algorithm, on the AMR problem, with an algorithm that constructs the AMR graph directly from the sentence tokens. Imitation learning has also been used successfully in other semantic parsing tasks (Vlachos and Clark, 2014; Berant and Liang, 2015).

In imitation learning approaches such as DAGGER the previous actions become features for classification learning. However the partial graphs in AMR parsing are rather complex to represent in this way, and combined with the finite amount of training data different actions can be chosen by the expert even though the feature representations for them can be very similar. These decisions appear as noisy outliers in classification learning. To control noise we experiment with the $\alpha$-bound discussed by Khardon and Wachman (2007), which excludes a training example from future training once it has been misclas- 


\begin{tabular}{|l|c|l|l|}
\hline Action Name & Param. & Pre-conditions & Outcome of action \\
\hline NextEdge & $l_{r}$ & $\beta$ non-empty & Set label of edge $\left(\sigma_{0}, \beta_{0}\right)$ to $l_{r}$. Pop $\beta_{0}$. \\
\hline NextNode & $l_{c}$ & $\beta$ empty & Set concept of node $\sigma_{0}$ to $l_{c}$. Pop $\sigma_{0}$, and initialise $\beta$. \\
\hline Swap & & $\beta$ non-empty & $\begin{array}{l}\text { Make } \beta_{0} \text { parent of } \sigma_{0} \text { (reverse edge) and its sub-graph. Pop } \beta_{0} \\
\text { and insert } \beta_{0} \text { as } \sigma_{1} \text {. }\end{array}$ \\
\hline ReplaceHead & $\kappa$ & $\beta$ non-empty & $\begin{array}{l}\text { Pop } \sigma_{0} \text { and delete it from the graph. Parents of } \sigma_{0} \text { become par- } \\
\text { ents of } \beta_{0} \text {. Other children of } \sigma_{0} \text { become children of } \beta_{0} \text {. Insert } \\
\beta_{0} \text { at the head of } \sigma \text { and re-initialise } \beta .\end{array}$ \\
\hline Reattach & $\begin{array}{l}\text { Pop } \beta_{0} \text { and delete edge }\left(\sigma_{0}, \beta_{0}\right) \text {. Attach } \beta_{0} \text { as a child of } \kappa . \text { If } \kappa \\
\text { has already been popped from } \sigma \text { then re-insert it as } \sigma_{1} \text {. }\end{array}$ \\
\hline DeleteNode & $l_{c}$ & $\beta$ empty; leaf $\sigma_{0}$ & Pop $\sigma_{0}$ and delete it from the graph. \\
\hline Insert & $l_{c}$ & & $\begin{array}{l}\text { Insert a new node } \delta \text { with AMR concept } l_{c} \text { as the parent of } \sigma_{0}, \\
\text { and insert } \delta \text { into } \sigma .\end{array}$ \\
\hline InsertBelow & $\kappa$ & Phase 2 & Insert a new node $\delta$ with AMR concept $l_{c}$ as a child of $\sigma_{0}$. \\
\hline Reentrance & $\omega$ & Phase 2 & Insert edge $\left(\sigma_{0}, \kappa\right)$ Then apply NextEdge action. \\
\hline Wikify & & See main text \\
\hline
\end{tabular}

Table 1: Action Space for the transition-based graph parsing algorithm

sified $\alpha$ times in training. Khardon and Wachman (2007) do not report any experimental results for this, and we are not aware of any previous use of this method.

\section{System description}

In the following subsections we focus on the differences from previous work and in particular that of Wang et al. (2015b) who introduced the transitionbased dependency-to-AMR paradigm we follow. We initialise the main algorithm with a stack of the nodes in the dependency tree, root node first. This stack is termed $\sigma$. A second stack, $\beta$ is initialised with all children of the top node in $\sigma$. The state at any time is described by $\sigma, \beta$, and the current graph (which starts as the dependency tree). Each action manipulates the top nodes in each stack, $\sigma_{0}$ and $\beta_{0}$. We reach a terminal state when $\sigma$ is empty. ${ }^{1}$

\subsection{The Expert Policy}

The expert policy used in training applies heuristic rules to determine the next action from a given state. It uses the training alignments to construct a mapping between nodes in the dependency tree, and nodes in the target AMR. Any unmapped nodes in the dependency tree will be deleted by the expert, and any unmapped nodes in the AMR graph will be

\footnotetext{
${ }^{1}$ Code available at hopshackle/dagger-AMR. bookmarks the version used.

https://github.com/ SemevalSubmission tag
}

inserted. All our experiments use node alignments from the system of Pourdamghani et al. (2014).

\subsection{Action Space}

Flanigan et al. (2014) and Wang et al. (2015b), both use AMR fragments as their smallest unit, which may consist of more than one AMR concept. Instead, we always work with the individual AMR nodes, and rely on Insert actions to learn how to build common fragments, such as country names. The main adaptations to the actions, summarised in Table 1, stem from this. NextNode and NextEdge form the core action set, labelling nodes and edges respectively without changing the graph structure. Swap, Reattach and ReplaceHead change this structure, but always retain a tree structure. ReplaceHead covers two distinct actions in Wang et al. (2015b); ReplaceHead and Merge. Their Merge action merges $\sigma_{0}$ and $\beta_{0}$ into a composite node; this is not required without composite nodes and retention of a 1:1 mapping between nodes and AMR concept. Unlike Wang et al. (2015b) we do not parameterise Swap or Reattach actions with a label. We leave that decision to a later NextEdge action. We permit a Reattach action to use parameter $\kappa$ equal to any node within six edges from $\sigma_{0}$, excluding any that would disconnect the graph or creating a cycle.

The Insert action inserts a new node as a parent of the current $\sigma_{0}$. Wang et al. (2015a) later introduced an 'Infer' action similar to our Insert action. Infer inserts an AMR concept node above the current node 
as Insert does, but is restricted to nodes that occur outside of AMR 'fragments', which continue to be the base building block.

Reentrance is the one action that will turn a Tree into a non-Tree. We only consider Reentrance actions during a second pass ("Phase Two") through the AMR graph once the first pass has reached a terminal state. In this second pass we consider each node as $\sigma_{0}$ in turn, and each nearby node as a possible $\kappa$ to insert a new edge $\left(\sigma_{0}, \kappa\right)$. We follow any Reentrance action with a NextEdge action to label the new arc. This approach simplifies the first pass, during which the graph is guaranteed to be a Tree. Reentrance makes only a small difference in the final F-Score, and was turned off for our final submission.

Also during Phase Two we decide whether to Wikify $\sigma_{0}$. This adds a new leaf node with a relation of wiki. There are three parameter values for $\omega$ that determine the wiki concept. In turn these use "-"; a concatenation of all child concepts in original word order; a dictionary look-up keyed on a concatenation of the child nodes if these were seen in the training data. An example of the third option is if a name node with a single child node of "Michael" is seen in the training data with a wiki relation of "Michael_Jackson". This wikification is held in the dictionary, and will be used for any name node with a single child node of "Michael" in test. If instead in test the name node had two children; "Michael", and "Jackson", then during $\omega$ would either add a wiki node of "-", or one of "Michael_Jackson" by concatenating the two child concepts in the order they appear in the sentence.

Figure 1 shows a parse of a sentence fragment. The current $\sigma_{0}$ node is shown dashed and in red. From the top the actions are Insert(dateentity); NextNode(WORD); NextEdge(year); second diagram; NextNode(WORD); ReplaceHead to remove "in"; third diagram; NextNode(WORD); NextEdge(mod); Reattach to move "date-entity"; fourth diagram; NextNode(VERB); ReplaceHead to remove "by"; NextEdge(ARG0); NextEdge(time); NextNode(strike-01).

Wang et al. (2015b) use all AMR concepts and relations that appear in the training set as possible parameters $\left(l_{c}\right.$ and $\left.l_{r}\right)$ if they appear in any sentence containing the same lemma as $\sigma_{0}$ and $\beta$. We reduce this to just concepts that have been aligned to the

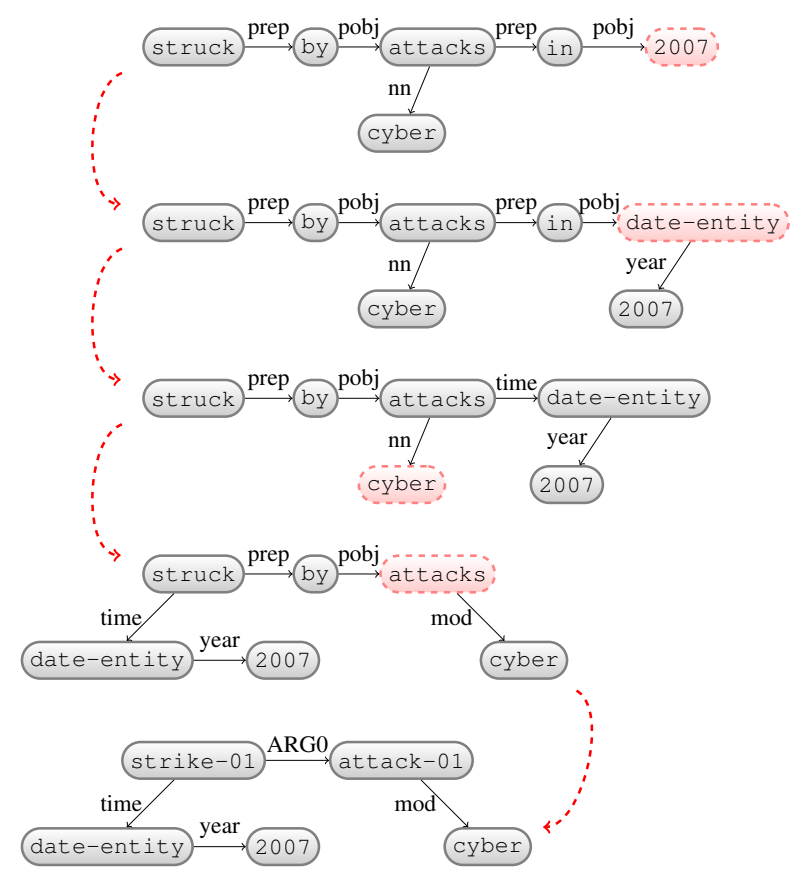

Figure 1: Example parse from dependency tree to AMR of the sentence fragment “... struck by cyber attacks in 2007."

current lemma. We initially run the expert policy over the training set, and track the AMR concept assigned for each lemma. These provide the possible $l_{c}$ that will be used for NextNode actions. Similarly we track the lemmas at head and tail of each expert-assigned AMR relation, and compile possible $l_{r}$ from these. There is no direct generalisation between different concepts and relations; so ARGO and $A R G 0-O f$ are independently learned relations for example, although they represent the same semantic relationship.

AMR concepts/relations will never be considered during test if they were not aligned to that lemma in the training data. To relax this restriction we allow $l_{c}$ to take the values WORD, LEMMA, VERB, which respectively use the word, lemma, or the lemma concatenated with ' -01 ' as the AMR concept. This is inspired by Werling et al. (2015), who use a similar set of actions in a concept identification phase. These options improve performance (by 0.5 to 1.0 points on a validation set) by generalising to unseen tokens in test data, which otherwise would have no mapped AMR concepts. For the $l_{c}$ parameters on Insert (InsertBelow) actions, we use all AMR concepts that the expert inserted above (below) any node in 
the training set with the same lemma as $\sigma_{0}$.

\subsection{Additional action constraints}

Transition-based parsing algorithms have classically relied on a fixed length of trajectory $T$ for guarantees on performance, or at least a bounded $T$ (Goldberg and Elhadad, 2010; Honnibal et al., 2013; Sartorio et al., 2013; McDonald and Nivre, 2007). In our approach $T$ is theoretically unbounded and the algorithm could Insert, or Reattach ad infinitum.

We impose constraints to prevent these situations. A Swap action cannot be applied to a previously Swapped edge; once a node has been moved by Reattach, then it cannot be Reattached again; an Insert action is only permissible if no previous Insert action has been used with that node as $\sigma_{0}$; an Insert action is not permissible if it would insert an AMR concept already in use as any of the parent, children, grand-parents or grand-children of $\sigma_{0}$.

Any action that would create a cycle is prohibited. We do not prevent duplication of argument relations so that a concept could have two outgoing ARG1 edges. We start with a fully connected graph (the dependency tree), and preserve full connectivity as none of the actions will disconnect a graph.

\subsection{Imitation Learning}

In the first iteration we only use the expert policy to generate a trajectory for each training sentence, and train a classifier $\pi_{1}$ from the collected data. At each step in the $i$ th iteration we randomly choose the expert (with probability $\beta_{i}$ ) or else $\pi_{i-1}$. The full set of collected data from all iterations is then used to train $\pi_{i-1}$ to obtain $\pi_{i} . \beta_{1}=1$ and we set $\beta_{i}=0.7 \beta_{i-1}$. Hence each iteration uses the expert policy less and less, with the training trajectories increasingly approximating the states the classifier would encounter without expert knowledge of the target. Formally the DAGGER algorithm is online (Ross et al., 2011), while we use it in a batch mode as described above. We use an averaged AROW classifier for all our experiments, with parameter $r=100$ (Crammer et al., 2009). After each batch DAGGER iteration we use 3 iterations of (on-line) AROW training using all the collected data.

As well as the $\alpha$-bound for noise reduction, we tried two additional modifications to DAGGER and AROW. Firstly, we considered reducing the num- ber of actions explored at each stage. The currently trained classifier evaluates all possible actions, and discards any that exceed the best scoring action by some threshold. Only this smaller set, plus the expert action, are included in the training example. This speeds up classifier training. In the first iteration we choose three to five actions randomly. Ross and Bagnell (2014) use a random set of exploratory actions in their AGGREVATE algorithm, but do not use the classifier to focus the exploration.

Secondly, we used only the smallest training sentences in the first iteration, as measured by number of AMR nodes. At each further iteration the AMR size threshold for the training set was increased. The motivation was to train the classifier on 'easy' sentences, before introducing more complex ones. We start with up to 30 nodes, and increase this by 10 each iteration. Rao et al. (2015) similarly use the smallest sentences for training, but do not increase the size threshold as training proceeds.

\subsection{Features}

All features used are detailed in Table 2, largely based on Wang et al. (2015b). All are 0-1 indicator functions. inserted is 1 if the node was inserted by the parser; $d l$ is the dependency label in the original dependency tree; ner the named entity tag; POS the part-of-speech tag; prefix is the string before the hyphen if word is hyphenated; suffix is the string after the hyphen; brown is the 100-class Brown cluster id with cuts at 4, 6, 10 and $20^{2}$; deleted is the lemma of any child node previously deleted by the parser; merged is the lemma of any node merged into this node by a ReplaceHead action; distance is the distance between the tokens in the sentence; path concatenates lemmas and dls between the tokens in the dependency tree; POSpath concatenates POS tags between the tokens; NERpath concatenates NER tags between the tokens.

The key differences to Wang et al. (2015b) are the inclusion of the brown, POSpath, NERpath, prefix and suffix feature types.

\subsection{Pre-processing}

Pre-processing steps on the training sentences were to: pass the full sentence through the Stanford De-

\footnotetext{
${ }^{2}$ From http://metaoptimize.com/projects / wordreprs / and the code of Liang (2005)
} 


\begin{tabular}{|l|l|}
\hline Context & Features \\
\hline$\sigma_{0}$ & lemma, dl, ner, POS, inserted, prefix, suffix, brown, deleted, lemma-dl \\
\hline$\sigma_{0 P}$ & inserted, lemma, brown \\
\hline$\sigma_{0 C}$ & label, ner, label-brown \\
\hline$\beta_{0}$ & inserted, POS, lemma, brown, ner, dl, prefix, suffix, merged \\
\hline$\kappa$ & ner, POS, lemma, brown, label \\
\hline$\sigma_{0} \rightarrow \beta_{0}$ & $\begin{array}{l}\text { label, path, lemma-path-lemma, POSpath, inserted-inserted, lemma-POS, POS-lemma, } \\
\text { dl-lemma, lemma-dl, lemma-label, label-lemma, ner-ner, distance }\end{array}$ \\
\hline$\beta_{0} \rightarrow \kappa$ & path, lemma-path-lemma, NERpath, POSpath, distance, lemma-POS, dl-lemma, ner-ner \\
\hline$\sigma_{0} \rightarrow \kappa$ & distance, lemma-path-lemma, brown-brown, NERpath, POSpath, lemma-dl, lemma-label \\
\hline$\sigma_{0 P} \rightarrow \sigma_{0}$ & label, POS-lemma, dl-lemma, ner-ner \\
\hline$\sigma_{0 P P} \rightarrow \sigma_{0 P} \rightarrow \sigma_{0}$ & lemma-lemma-lemma \\
\hline$\sigma_{0} \rightarrow \sigma_{0 C}$ & POS-lemma, lemma-POS, dl-lemma, ner-ner \\
\hline
\end{tabular}

Table 2: Features used by context. $\sigma_{0 P}$ is the parent of $\sigma_{0}, \sigma_{0 P P}$ the parent of $\sigma_{0 P}$, and $\sigma_{0 C}$ a child of $\sigma_{0}$.

pendency Parser v3.3.1 to construct a dependency tree (Manning et al., 2014); remove punctuation tokens; " " characters are treated as token separators, but hyphenated words are kept as single tokens; simple regex expressions are applied to find common date formats, and convert these to numeric sequences (e.g. 03-Jan-72 to 31 1972); similar regex conversions of common numeric expressions e.g. "two thousand" becomes "2000". The parser was then able to learn to construct date-entity, temporal-quantity and similar AMR moieties.

\section{Results}

In all experiments, the training data was the union of all training and dev sets in the task data, and the union of all test sets was used for validation. Table 3 shows the F-Score of the validation set with and without the Reentrance action (Reent, NoR), and with combinations of reduced search (Red), and incremental data (Inc). All of these give the same result of 0.65 to $2 \mathrm{dp}$, and the bold entry was submitted. The Baseline entry uses only a single iteration, and the DAGGER experiments report the highest FScore achieved over 10 iterations (usually reached between the 3rd to 5th iterations).

The reduced information in the first iterations for incremental data and reduced search lead to lower initial performance here, but they still achieve the 0.65 result in time and each iteration is faster. DAGGER provides a gain of 0.6 and 1.1 points of F-Score for experiments with and without Reentrance.

Table 4 shows that the $\alpha$-bound helps signifi-
Table 3: F-Score results on validation set $(\alpha=1)$.

\begin{tabular}{|l|c|c|}
\hline Parameter settings & NoR & Reent. \\
\hline Baseline & 0.642 & 0.640 \\
\hline DAGGER & 0.648 & 0.651 \\
\hline DAGGER \& Inc & 0.653 & 0.655 \\
\hline DAGGER \& Red & 0.648 & 0.653 \\
\hline DAGGER \& Inc \& Red & $\mathbf{0 . 6 5 1}$ & 0.649 \\
\hline
\end{tabular}

cantly, with a gain of 1.8 points of F-Score in this example. We found consistently that the most extreme setting of $\alpha=1$ worked best.

Table 4: Alpha-bound results with DAGGER, Inc and Red

\begin{tabular}{|c|c|}
\hline Alpha-bound & F-Score \\
\hline 1 & 0.655 \\
\hline 2 & 0.649 \\
\hline None & 0.637 \\
\hline
\end{tabular}

\section{Conclusion}

Imitation Learning algorithms like DAGGER help in the AMR task, as in other structured prediction problems. Performance is improved using the $\alpha$-bound to reduce the impact of noise in the training examples, and future work could investigate the impact in similar tasks.

The reduction in search space and incremental growth of the training set do not have a significant impact on the results. The speed improvements they provide make little difference here, but could benefit more computationally demanding loss functions than the 0-1 expert loss used in DAGGER. 


\section{Acknowledgments}

Andreas Vlachos is supported by the EPSRC grant Diligent (EP/M005429/1) and Jason Naradowsky by a Google Focused Research award.

\section{References}

Laura Banarescu, Claire Bonial, Shu Cai, Madalina Georgescu, Kira Griffitt, Ulf Hermjakob, Kevin Knight, Phillip Koehn, Martha Palmer, and Nathan Schneider. 2013. Abstract Meaning Representation for Sembanking. Proceedings of the 7th Lingustic Annotation Workshop \& Interoperability with Discourse, pages 178-186.

Jonathan Berant and Percy Liang. 2015. Imitation learning of agenda-based semantic parsers. Transactions of the Association for Computational Linguistics (TACL), 3:545-558.

Koby Crammer, Alex Kulesza, and Mark Dredze. 2009. Adaptive regularization of weight vectors. In $A d$ vances in neural information processing systems, pages 414-422.

Jeffrey Flanigan, Sam Thomson, Jaime Carbonell, Chris Dyer, and Noah A Smith. 2014. A discriminative graph-based parser for the abstract meaning representation.

Yoav Goldberg and Michael Elhadad. 2010. An efficient algorithm for easy-first non-directional dependency parsing. In Human Language Technologies: The 2010 Annual Conference of the North American Chapter of the Association for Computational Linguistics, pages 742-750. Association for Computational Linguistics.

Matthew Honnibal, Yoav Goldberg, and Mark Johnson. 2013. A non-monotonic arc-eager transition system for dependency parsing. In Proceedings of the Seventeenth Conference on Computational Natural Language Learning, pages 163-172. Citeseer.

Roni Khardon and Gabriel Wachman. 2007. Noise tolerant variants of the perceptron algorithm. The journal of machine learning research, 8:227-248.

Percy Liang. 2005. Semi-supervised learning for natural language. Ph.D. thesis, Citeseer.

Christopher D. Manning, Mihai Surdeanu, John Bauer, Jenny Finkel, Steven J. Bethard, and David McClosky. 2014. The Stanford CoreNLP natural language processing toolkit. In Proceedings of 52nd Annual Meeting of the Association for Computational Linguistics: System Demonstrations, pages 55-60.

Ryan T McDonald and Joakim Nivre. 2007. Characterizing the errors of data-driven dependency parsing models. In EMNLP-CoNLL, pages 122-131.
Nima Pourdamghani, Yang Gao, Ulf Hermjakob, and Kevin Knight. 2014. Aligning english strings with abstract meaning representation graphs. In Proceedings of the 2014 Conference on Empirical Methods in Natural Language Processing (EMNLP), pages 425429.

Sudha Rao, Yogarshi Vyas, Hal Daume III, and Philip Resnik. 2015. Parser for abstract meaning representation using learning to search. arXiv preprint arXiv: 1510.07586.

Stephane Ross and J Andrew Bagnell. 2014. Reinforcement and imitation learning via interactive no-regret learning. arXiv preprint arXiv:1406.5979.

Stéphane Ross, Geoffrey J Gordon, and J Andrew Bagnell. 2011. A reduction of imitation learning and structured prediction to no-regret online learning.

Francesco Sartorio, Giorgio Satta, and Joakim Nivre. 2013. A transition-based dependency parser using a dynamic parsing strategy. In ACL (1), pages 135-144.

Andreas Vlachos and Stephen Clark. 2014. A new corpus and imitation learning framework for contextdependent semantic parsing. Transactions of the Association for Computational Linguistics, 2:547-559.

Chuan Wang, Nianwen Xue, and Sameer Pradhan. 2015a. Boosting transition-based amr parsing with refined actions and auxiliary analyzers. In Proceedings of the 53rd Annual Meeting of the Association for Computational Linguistics and the 7th International Joint Conference on Natural Language Processing (Volume 2: Short Papers), pages 857-862, Beijing, China, July. Association for Computational Linguistics.

Chuan Wang, Nianwen Xue, and Sameer Pradhan. 2015b. A transition-based algorithm for amr parsing. North American Association for Computational Linguistics, Denver, Colorado.

Keenon Werling, Gabor Angeli, and Christopher D. Manning. 2015. Robust subgraph generation improves abstract meaning representation parsing. In Proceedings of the 53rd Annual Meeting of the Association for Computational Linguistics and the 7th International Joint Conference on Natural Language Processing (Volume 1: Long Papers), pages 982-991, Beijing, China, July. Association for Computational Linguistics. 